$\underline{\underline{p}}=\mathrm{Z}$

\title{
On fixing the magnitudes of gravitational constant and strong coupling constant
}

\author{
U. V.S. Seshavatharam ${ }^{1 *}$, S. Lakshminarayana ${ }^{2}$ \\ ${ }^{1}$ Honorary faculty, I-SERVE, Alakapuri, Hyderabad-35,Ttelengana, India \\ ${ }^{2}$ Dept. of Nuclear Physics, Andhra University, Visakhapatnam-03,AP, India \\ *Corresponding author E-mail: seshavatharam.uvs@gmail.com
}

\begin{abstract}
In the earlier published papers the authors suggested that, "Magnitude of the unified force can be assumed to be equal to the classical or astrophysical force limit $\left(c^{4} / G\right)$. Strength of any interaction can be defined as the ratio of the operating force magnitude and the magnitude of $\left(c^{4} / G\right)$. If strength of the Schwarzschild interaction is assumed to be unity, then weak interaction strength seems to be 'squared Avogadro number' times less than the Schwarzschild interaction. The characteristic atomic force can be represented by $\left(c^{4} / N_{A}^{2} G\right)$ ". Thinking in this way, atomic gravitational constant can be expressed as $G_{A} \cong N_{A}^{2} G$. With current atomic physical constants and with the assumed two new grand unified back ground numbers $x \cong 38.72479081$ and $\mathrm{y} \cong 47.41543166$, analytically - value of $G$ can be fixed for 10 digits and can be verified. Inverse of the strong coupling constant can be considered as the 'natural logarithm of square root of ratio of gravitational and electromagnetic force ratio of down quark mass where the operating gravitational constant is squared Avogadro number times the gravitational constant'. Finally an attempt is made to fit and understand the mystery of Up and Down quarks, nuclear stability, and nuclear binding energy. For medium and heavy atomic nuclides, at the stable mass number, nuclear binding energy seems to be equal to the sum of rest energy of $2 Z$ up quarks and $Z$ down quarks.
\end{abstract}

Keywords: Gravitational constant; Schwarzschild's interaction; Astrophysical force limit; Avogadro number; Particle rest masses; Strong interaction; nuclear binding energy; and Electron's $\left(n^{2}\right)$ quantum states.

\section{Introduction}

From final unification point of view, it is very much essential to couple the universal gravitational constant with the elementary physical constants. Then only the essence of unification can be understood. So far scientists proposed several interesting models (P. A. M. Dirac 1937), (Witten, Edward 1981), (David Gross 2005), (Abdus Salam 1981), (Salam A. \& Sivaram C 1993), (Recami E 1994), (Dine, Michael 2007), (Roberto Onofrio 2013). In this context, readers may go through the authors published papers (U. V. S. Seshavatharam \& S. Lakshminarayana 2014, 2013, 2011). By introducing two new back ground unified numbers $(x, y)$, in the published paper the authors expressed their views (U. V. S. Seshavatharam \& S. Lakshminarayana 2014) on final unification and proposed three characteristic relations for connecting, fitting and verifying the Newtonian gravitational constant in a unified approach via the Avogadro number $N_{A}$. In this paper, the topics covered and reviewed are: Schwarzschild interaction strength, meaning of strength of interaction in atomic physics, significance of Avogadro number, fitting of the gravitational constant, muon an tau rest masses, strong coupling constant, fine structure ratio, reduced Planck's constant, rest masses of Up \&
Down quarks, Nucleon rest masses, rms radius of proton, nuclear charge radius, nuclear stability, nuclear binding energy and unified atomic mass unit. Important points can be expressed as follows.

1) In any inverse square law of force, system is sustained only by means of the central attractive force and it is the root cause of revolving body's angular momentum. If it is confirmed that, revolving body's angular momentum is discrete, then it is a clear indication of the discrete nature of the central force acting on the revolving body. If one is willing to think in this direction, the historical mystery of Bohr's discrete atomic structure and discrete angular momentum can be understood.

2) Note that, as per the basic concepts of final unification, there exists a fundamental unified force from which all the observed forces emerged. If so, magnitude of the unified force can be assumed to be equal to the astrophysical force limit $\left(c^{4} / G\right)$. Note that, magnitude of the radial inward force acting on any black hole surface (U. V. S. Seshavatharam \& S. Lakshminarayana 2014) is of the order of $\left(c^{4} / G\right)$. 


\section{The classical limits of force and power}

To unify cosmology, quantum mechanics and the four observed fundamental cosmological interactions certainly a 'unified force' is required. In this connection $\left(c^{4} / G\right)$ can be considered as the classical force or astrophysical force limit. Similarly $\left(c^{5} / G\right)$ can be considered as the classical power limit. If it is true that $c$ and $G$ are fundamental physical constants in physics, then $\left(c^{4} / G\right)$ and $\left(c^{5} / G\right)$ can also be considered as fundamental compound physical constants. These classical limits are more powerful than the Uncertainty limit. Without considering the current notion of black hole physics, Schwarzschild radius of black hole (Roger Penrose 1996), (Subrahmanyan Chandrasekhar 1983) can be understood with the characteristic astrophysical limiting force of magnitude $\left(c^{4} / G\right)$. Note that by considering $\left(c^{4} / G\right)$, the famous Planck mass can be obtained very easily.

\subsection{Simple applications of $\left(c^{4} / G\right)$}

a) Magnitude of force of attraction or repulsion between any two charged particles never exceeds $\left(c^{4} / G\right)$.

b) Magnitude of gravitational force of attraction between any two massive bodies never exceeds $\left(c^{4} / G\right)$.

c) Magnitude of mechanical force on a revolving/rotating body never exceeds $\left(c^{4} / G\right)$.

d) Magnitude of electromagnetic force on a revolving body never exceeds $\left(c^{4} / G\right)$.

\subsection{Simple applications of $\left(c^{5} / G\right)$}

a) Mechanical power never exceeds $\left(c^{5} / G\right)$

b) Electromagnetic power never exceeds $\left(c^{5} / G\right)$

c) Thermal radiation power never exceeds $\left(c^{5} / G\right)$

d) Gravitational radiation power never exceeds $\left(c^{5} / G\right)$

\section{Understanding the role of $\left(c^{4} / G\right)$ in black hole formation and Planck mass generation}

\subsection{Schwarzschild radius of a black hole}

The four basic physical properties of a rotating black hole are its mass, size, angular velocity and temperature. Without going deep into the mathematics of black hole physics in this section an attempt is made to understand the Schwarzschild radius of a black hole. In all directions, if a force of magnitude $\left(c^{4} / G\right)$ acts on the mass-energy content of the assumed celestial body it approaches a minimum radius of $\left(G M / c^{2}\right)$ in the following way. Origin of the force $\left(c^{4} / G\right)$ may be due to self-weight or internal attraction or external compression or something else.

$$
R_{\min } \cong \frac{M c^{2}}{\left(c^{4} / G\right)} \cong \frac{G M}{c^{2}}
$$

If no force (of zero magnitude) acts on the mass content $M$ of the assumed massive body, its radius becomes infinity. With reference to the average magnitude of $\left(0, \frac{c^{4}}{G}\right) \cong \frac{c^{4}}{2 G}$, the presently believed Schwarzschild radius can be obtained as

$$
(R)_{a v e} \cong \frac{M c^{2}}{\left(c^{4} / 2 G\right)} \cong \frac{2 G M}{c^{2}}
$$

This proposal is very simple and seems to be different from the existing concepts and may be a unified form of the Newton's law of gravity, Special theory of relativity and General theory of relativity.

\subsection{To derive the Planck mass}

So far no theoretical model proposed a derivation for the Planck mass. To derive the Planck mass the following two conditions can be given a chance.

Assuming that gravitational force of attraction between two Planck particles of mass $\left(M_{P}\right)$ separated by a minimum distance $\left(r_{\min }\right)$ be,

$\left[\frac{G M_{P} M_{P}}{r_{\min }^{2}}\right] \equiv\left(\frac{c^{4}}{G}\right)$

With reference to wave mechanics, let

$2 \pi \cdot r_{\min } \cong \lambda_{P}=\left[\frac{h}{c \cdot M_{P}}\right]$

Here, $\lambda_{P}$ represents the wavelength associated with the Planck mass. With these two assumed conditions Planck mass can be obtained as follows.

$M_{P}=\sqrt{\frac{h c}{2 \pi G}} \cong \sqrt{\frac{\hbar c}{G}}$

\subsection{Understanding the strength of any interaction}

From the above relations it is reasonable to say that,

1) If it is true that $c$ and $G$ are fundamental physical constants, then $\left(c^{4} / G\right)$ can be considered as a fundamental compound constant related to a characteristic limiting force.

2) Black holes are the ultimate state of matter's geometric structure.

3) Magnitude of the operating force at the black hole surface is the order of $\left(c^{4} / G\right)$.

4) Gravitational interaction taking place at black holes can be called as 'Schwarzschild interaction'.

5) Strength of 'Schwarzschild interaction' can be assumed to be unity.

6) Strength of any other interaction can be defined as the ratio of operating force magnitude and the classical or astrophysical force magnitude $\left(c^{4} / G\right)$. 
7) If one is willing to represent the magnitude of the operating force as a fraction of $\left(c^{4} / G\right)$ i.e $X$ times of $\left(c^{4} / G\right)$, where $X=1$, then

$$
\frac{X \text { times of }\left(c^{4} / G\right)}{\left(c^{4} / G\right)} \cong X \rightarrow \text { Effective } G \Rightarrow \frac{G}{X}
$$

If $X$ is very small, $\frac{1}{X}$ becomes very large. In this way, $X$ can be called as the strength of interaction. Clearly speaking, strength of any interaction is $\frac{1}{X}$ times less than the 'Schwarzschild interaction' and effective $G$ becomes $\frac{G}{X}$.

\section{Basic concepts and relations final unifica- tion}

The following concepts and relations can be given a chance in final unification program.

1) With reference to the elementary charge and with mass similar to the Planck mass, a new mass unit can be constructed in the following way.

$$
\left.\begin{array}{l}
\left(M_{S}\right)^{ \pm} \cong \sqrt{\frac{e^{2}}{4 \pi \varepsilon_{0} G}} \cong 1.859272 \times 10^{-9} \mathrm{~kg} \\
M_{S} c^{2} \cong \sqrt{\frac{e^{2} c^{4}}{4 \pi \varepsilon_{0} G}} \cong 1.042975 \times 10^{18} \mathrm{GeV}
\end{array}\right\}
$$

It can be called as the Stoney mass (G. J. Stoney, 1881). It is well known that $e, c, G$ play a vital role in fundamental physics. With these 3 constants, space-time curvature concepts at a charged particle surface can be studied. It was first introduced by the physicist George Johnstone Stoney. He is most famous for introducing the term 'electron' as the 'fundamental unit quantity of electricity'. In unification program, with this mass unit and with a suitable proportionality ratio- characteristic mass of any elementary charge can be generated.

2) Avogadro number is an absolute number and it is having no units like 'per mole'.

3) Atomic interaction strength is $N_{A}^{2}$ times less than the Schwarzschild interaction and hence atomic gravitational constant can be expressed as:

$G_{A} \cong N_{A}^{2} G$

4) Similar to the classical force limit $\left(c^{4} / G\right)$, in atomic system there exists a characteristic force of magnitude:

$F_{X} \cong\left(1 / N_{A}^{2}\right)\left(c^{4} / G\right) \cong\left(c^{4} / N_{A}^{2} G\right)$

5) Independent of system of units and without considering the Avogadro number, unified atomic mass unit (P.J. Mohr et al 2010), (B. Andreas et al 2011), (B P Leonard 2007),(K.A. Olive et al 2014) can be fitted as follows.

$$
m_{u} c^{2} \cong\left(\sqrt{m_{n} m_{p}} c^{2}-B_{a}\right)+m_{e} c^{2}
$$

Where $m_{u}$ is the unified atomic mass unit and $B_{a}$ is the average binding energy per nucleon. If $B_{a} \cong(7.90$ to 8.0$) \mathrm{MeV}$, obtained magnitude of $m_{u} \cong 931.4295$ to $931.5295 \mathrm{MeV} / \mathrm{c}^{2}$. Thus it can be suggested that, accuracy of $m_{u}$ depends only on the accurate 'average binding energy per nucleon'.

\subsection{Semi empirical applications of $(x, y)$ :}

There exist two new numbers $(x, y)$. they can be called as the 'primordial unified back ground numbers'. They can also be called as the 'back ground analytical numbers' using by which micromacro physical constants can be interlinked qualitatively and quantitatively.

Application-1: Rest masses of electron and proton

Electron rest mass can be expressed in the following way.

$m_{e} \cong x^{\frac{1}{2}} y \sqrt{\frac{e^{2}}{4 \pi \varepsilon_{0} G_{A}}}$

With $(x, y)$, proton rest mass can be expressed in the following way.

$m_{p} \cong x^{\frac{3}{2}} y^{2} \sqrt{\frac{e^{2}}{4 \pi \varepsilon_{0} G_{A}}}$

Thus,

$\frac{m_{p}}{m_{e}} \cong x y$

Application-2: Rest masses of muon and tau

Let, $\beta \cong x^{\frac{1}{2}} y$

Where $\beta$ can be called as the electron mass index. It can be estimated as:

$\beta \cong x^{\frac{1}{2}} y \cong \sqrt{\frac{4 \pi \varepsilon_{0} N_{A}^{2} G m_{e}^{2}}{e^{2}}} \cong 295.0509223$

With this number $\beta$, electron, muon and tau rest masses can be fitted with the semi empirical relation.

$\left.\begin{array}{l}\left(m_{\text {lepton }}\right)_{n} c^{2} \cong\left[\beta^{3}+\left(n^{2} \beta\right)^{n} \sqrt{N_{A}}\right]^{\frac{1}{3}} \sqrt{\frac{e^{2} F_{X}}{4 \pi \varepsilon_{0}}} \\ \cong\left[\beta^{3}+\left(n^{2} \beta\right)^{n} \sqrt{N_{A}}\right]^{\frac{1}{3}} 0.001731 \mathrm{MeV}\end{array}\right\}$

Where $n=0,1,2$. Obtained rest energies are $0.511 \mathrm{MeV}, 105.95$ $\mathrm{MeV}$ and $1777.4 \mathrm{MeV}$ respectively (K.A. Olive et al 2014). New heavy charged lepton at $n=3$ may be predicted close to 42262 $\mathrm{MeV}$.

Application-3: The reduced Planck's constant and the rms radius of proton

From above relations, 


$$
\left.\begin{array}{l}
x \cong\left(\frac{1}{\beta} \frac{m_{p}}{m_{e}}\right)^{2} \cong 38.72787108 \\
y \cong\left(\frac{1}{x} \frac{m_{p}}{m_{e}}\right) \cong 47.41166036
\end{array}\right\}
$$

If so, Reduced Planck's constant can be expressed in the following way.

$$
\left(e^{x}\right)^{-\frac{1}{6}}\left(\frac{G_{A} m_{e}^{2}}{c}\right) \cong \hbar \cong 1.053946635 \times 10^{-34} \mathrm{~J} . \mathrm{sec}
$$

Characteristic nuclear radii like rms radius of proton (P.J. Mohr et al 2010), (Geiger H \& Marsden. E 1909), (Michael O. Distler et al 2011), (Roberto Onofrio 2013) nuclear charge radius etc can be expressed in the following way.

$$
\left.\begin{array}{l}
\left(e^{x}\right)^{-\frac{1}{2}}\left(\frac{G_{A}{ }^{m} p}{c^{2}}\right) \cong 1.753816617 \times 10^{-15} \mathrm{~m} \\
\frac{1}{2}\left(e^{x}\right)^{-\frac{1}{2}}\left(\frac{G_{A}{ }^{m} p}{c^{2}}\right) \cong 0.8769083083 \times 10^{-15} \mathrm{~m} \\
\sqrt{\frac{1}{2}}\left(e^{x}\right)^{-\frac{1}{2}}\left(\frac{G_{A}{ }^{m} p}{c^{2}}\right) \cong 1.240135623 \times 10^{-15} \mathrm{~m}
\end{array}\right\}
$$

If so, it is possible to show that,

$$
\hbar \cong\left(\frac{2 R_{p^{c}}{ }^{2}}{G_{A} m_{p}}\right)^{\frac{1}{3}}\left(\frac{G_{A} m_{e}^{2}}{c}\right)
$$

\section{Application-4: To fit and verify the gravitational constant}

In astronomy, the only one available characteristic empirical physical constant is the gravitational constant. Its value has been measured in the lab only within a range of $1 \mathrm{~cm}$ to a few meters. Until one measures the value of the gravitational constant with microscopic physical constants, the debate of final unification cannot be stopped up. In this context, G. Rosi et al say (G. Rosi et al 2014): "There is no definitive relationship between $G$ and the other fundamental constants, and there is no theoretical prediction for its value, against which to test experimental results. Improving the precision with which we know $G$ has not only a pure metrological interest, but is also important because of the key role that $G$ has in theories of gravitation, cosmology, particle physics and astrophysics and in geophysical models". In general, 'Unification' means:

a) Understanding the origin of the rest mass of atomic elementary particles.

b) Finding and understanding the critical compositeness of the elementary physical constants.

c) Minimizing the number of elementary physical constants.

d) Merging different branches of physics with possible and suitable physical concepts.

Considering the proposed concepts and relations accurate values of Gravitational constant (L.L. Williams 2009), (George T Gillies 1997), (J Stuhler et al 2003), (Terry Quinn 2013), (J. B. Fixler et al 2007), (Brandenburg, J.E 1992), (Jun Luo and Zhong-Kun Hu 2000), (St. Schlamminger et al 2002) and Avogadro number can be estimated from elementary atomic physical constants. For the time being (i.e until a perfect model is developed), if one is willing to consider the revolving electron's angular momentum as a compound physical constant and depends on the proton-electron rest masses, characteristic nuclear charge radius and the proposed discrete force $\left(c^{4} / N_{A}^{2} G\right)$, it paves a path for coupling and interconnecting the micro-macro elementary physical constants in a consistent manner. Thus it is possible to couple Avogadro number and Gravitational constant in the following way.

$x \cong \ln \left(\frac{G_{A} m_{e}^{2}}{\hbar c}\right)^{6}$

$y \cong\left(\frac{m_{e}}{m_{p}}\right) \frac{4 \pi \varepsilon_{0} G_{A} m_{e}^{2}}{e^{2}}$

$x y-\left(\frac{m_{p}}{m_{e}}\right) \cong 0$

By considering

$N_{A} \cong 6.022141293 \times 10^{23}$

$m_{e} \cong 9.109382914 \times 10^{-31} \mathrm{~kg}$

$m_{p} \cong 1.672621777 \times 10^{-27} \mathrm{~kg}$

$\mathrm{h} \cong 1.054571726 \times 10^{-34} \mathrm{~J} . \mathrm{sec}$

$c \cong 2.99792458 \times 10^{8} \mathrm{~m} / \mathrm{sec}$

$\mathrm{e} \cong 1.602176565 \times 10^{-19} \mathrm{C}$

$\varepsilon_{0} \cong 8.854187817 \times 10^{-12} \mathrm{~J} . \mathrm{m}$

and by assuming,

$G \cong 6.674378868 \times 10^{-11} \mathrm{~m}^{3} \mathrm{~kg}^{-1} \mathrm{sec}^{-2}$

obtained values of $x$ and $y$ are,

$x \cong 38.72479081$ and $\mathrm{y} \cong 47.41543166$

Thus relations $(20,21$ and 22) can be considered as the 3 characteristic semi empirical unified relations. This assumed value of $G$ may not be absolute but can be given some consideration in unification program for further analysis This entire procedure depends on the two proposed new numbers $(x, \mathrm{y})$ and needs further research. So far there is no verifying procedure for the measured or estimated magnitude of $G$. with this kind of procedure, like other physical constants, value of $G$ can be fixed for 10 digits.

Application-5: Strong coupling constant, Up and Down quarks and nuclear binding energy

Inverse of the strong coupling constant can be fitted as follows (A.V. Manohar and C.T. Sachrajdahttp 2014):

$$
\begin{aligned}
& \frac{1}{\alpha_{s}} \cong y-x \cong 8.69064085 \\
& \rightarrow \alpha_{s} \cong 0.115066313
\end{aligned}
$$

Now Down quark mass can be expressed as follows (Halzen, F.; Martin, A. D 1984)

$$
\begin{aligned}
m_{d} c^{2} \cong \exp \left(\frac{1}{\alpha_{s}}\right) \sqrt{\frac{e^{2} F_{X}}{4 \pi \varepsilon_{0}}} \cong \exp \left(\frac{1}{\alpha_{s}}\right) 0.001731 \mathrm{MeV} \\
\cong 10.3 \mathrm{MeV}
\end{aligned}
$$

$$
\left(\frac{1}{\alpha_{S}}\right) \cong \ln \sqrt{\frac{4 \pi \varepsilon_{0} G_{A} m_{d}^{2}}{e^{2}}}
$$


Ratio of Up and down quark masses can be guessed as follows.

$$
\left.\begin{array}{c}
\left(\frac{m_{d} c^{2}}{m_{u} c^{2}}\right) \cong \ln \left(\frac{1}{\alpha_{s}}\right) \cong 2.1622467 \\
\left(\frac{m_{u} c^{2}}{m_{d} c^{2}}\right) \cong\left[\ln \left(\frac{1}{\alpha_{s}}\right)\right]^{-1} \cong 0.462482
\end{array}\right\}
$$

Thus Up quark mass can be fitted as follows.

$$
m_{u} c^{2} \cong\left[\ln \left(\frac{1}{\alpha_{s}}\right)\right]^{-1} \cong 4.764 \mathrm{MeV}
$$

Note that, these proposed Up and Down quark masses are roughly 2.20 times higher than the current estimates and their proposed mass ratio is matching with the current estimates. In a super symmetric approach, neutron and proton mass difference can be expressed as follows.

$$
\left(m_{n}-m_{p}\right) c^{2} \cong \frac{\left(m_{e} m_{u} m_{d}\right)^{1 / 3} c^{2}}{\psi} \cong \frac{\left(m_{e} m_{u} m_{d}\right)^{1 / 3} c^{2}}{2.26}
$$

Where $\psi \cong 2.26$ can be considered as the super symmetric fermion-boson mass ratio (U. V. S. Seshavatharam \& S. Lakshminarayana 2010, 2011).

With Up and Down quark masses nuclear binding energy (Chowdhury, P.R. et al 2005), (W.D. Myers \& W.J. Swiatecki 1994), (G. Audi \& A.H. Wapstra 1993) can be fitted as follows. Step-1: To fit the stable mass number of $Z$

$$
A_{S} \cong 2 Z+\left(\frac{m_{u}}{m_{d}}\right)\left(Z \alpha_{s}\right)^{2}
$$

Step-2: To fit the nuclear binding energy at stable mass number of Z

$$
(B)_{A_{S}} \cong k\left(2 Z m_{u} c^{2}+Z m_{d} c^{2}\right)
$$

Where, $\left\{\begin{array}{l}\text { For } Z>=30, k \cong 1.0 \text { and } \\ \text { for } Z<30, k=(Z / 30)^{1 / 6}\end{array}\right\}$

See table-1 for the estimated nuclear binding energy near to the stable mass number. Considering even-odd corrections on the estimated stable mass number and

With further research, data accuracy can be improved. From the data it is very clear to say that:

1) At the stable mass number, nuclear binding energy seems to be equal to the sum of rest energy of $2 Z$ up quarks and $Z$ down quarks.

2) As per the quark theory proton constitutes two Up quarks and one Down quark. Hence it can be guessed that, near to stable mass number, nuclear binding energy seems to depend only on the proton number.

Step-3: To fit the nuclear binding energy above and below the

\begin{tabular}{|c|c|c|c|}
\hline $\begin{array}{l}\text { Proton } \\
\text { number }\end{array}$ & $\begin{array}{l}\text { Estimated sta- } \\
\text { ble mass num- } \\
\text { ber } \\
A_{S}\end{array}$ & $\begin{array}{l}\text { Estimated } \\
\text { value of } k\end{array}$ & $\begin{array}{l}\text { Binding energy in } \mathrm{MeV} \\
\text { (near to stable mass num- } \\
\text { ber) }\end{array}$ \\
\hline 2 & 4 & 0.6368 & 25.3 \\
\hline 3 & 6 & 0.6813 & 40.5 \\
\hline 4 & 8 & 0.7148 & 56.7 \\
\hline 5 & 10 & 0.7418 & 73.5 \\
\hline 6 & 12 & 0.7647 & 91.0 \\
\hline 7 & 14 & 0.7846 & 108.9 \\
\hline 8 & 16 & 0.8023 & 127.3 \\
\hline 9 & 18 & 0.8182 & 146.0 \\
\hline 10 & 21 & 0.8327 & 165.1 \\
\hline 11 & 23 & 0.8460 & 184.5 \\
\hline 12 & 25 & 0.8584 & 204.2 \\
\hline 13 & 27 & 0.8699 & 224.2 \\
\hline 14 & 29 & 0.8807 & 244.5 \\
\hline 15 & 31 & 0.8909 & 265.0 \\
\hline 16 & 34 & 0.9005 & 285.7 \\
\hline 17 & 36 & 0.9097 & 306.6 \\
\hline 18 & 38 & 0.9184 & 327.8 \\
\hline 19 & 40 & 0.9267 & 349.1 \\
\hline 20 & 42 & 0.9347 & 370.6 \\
\hline 21 & 45 & 0.9423 & 392.4 \\
\hline 22 & 47 & 0.9496 & 414.2 \\
\hline 23 & 49 & 0.9567 & 436.3 \\
\hline 24 & 52 & 0.9635 & 458.5 \\
\hline 25 & 54 & 0.9701 & 480.9 \\
\hline 26 & 56 & 0.9764 & 503.4 \\
\hline 27 & 58 & 0.9826 & 526.0 \\
\hline 28 & 61 & 0.9886 & 548.8 \\
\hline 29 & 63 & 0.9944 & 571.8 \\
\hline 30 & 66 & 1.0000 & 594.8 \\
\hline 31 & 68 & 1.0000 & 614.7 \\
\hline 32 & 70 & 1.0000 & 634.5 \\
\hline 33 & 73 & 1.0000 & 654.3 \\
\hline 34 & 75 & 1.0000 & 674.2 \\
\hline 35 & 78 & 1.0000 & 694.0 \\
\hline 36 & 80 & 1.0000 & 713.8 \\
\hline 37 & 82 & 1.0000 & 733.6 \\
\hline 38 & 85 & 1.0000 & 753.5 \\
\hline 39 & 87 & 1.0000 & 773.3 \\
\hline 40 & 90 & 1.0000 & 793.1 \\
\hline 41 & 92 & 1.0000 & 812.9 \\
\hline 42 & 95 & 1.0000 & 832.8 \\
\hline 43 & 97 & 1.0000 & 852.6 \\
\hline 44 & 100 & 1.0000 & 872.4 \\
\hline 45 & 102 & 1.0000 & 892.3 \\
\hline 46 & 105 & 1.0000 & 912.1 \\
\hline 47 & 108 & 1.0000 & 931.9 \\
\hline 48 & 110 & 1.0000 & 951.7 \\
\hline 49 & 113 & 1.0000 & 971.6 \\
\hline 50 & 115 & 1.0000 & 991.4 \\
\hline 51 & 118 & 1.0000 & 1011.2 \\
\hline 52 & 121 & 1.0000 & 1031.1 \\
\hline 53 & 123 & 1.0000 & 1050.9 \\
\hline 54 & 126 & 1.0000 & 1070.7 \\
\hline 55 & 129 & 1.0000 & 1090.5 \\
\hline 56 & 131 & 1.0000 & 1110.4 \\
\hline 57 & 134 & 1.0000 & 1130.2 \\
\hline 58 & 137 & 1.0000 & 1150.0 \\
\hline 59 & 139 & 1.0000 & 1169.9 \\
\hline 60 & 142 & 1.0000 & 1189.7 \\
\hline 61 & 145 & 1.0000 & 1209.5 \\
\hline 62 & 148 & 1.0000 & 1229.3 \\
\hline 63 & 150 & 1.0000 & 1249.2 \\
\hline 64 & 153 & 1.0000 & 1269.0 \\
\hline 65 & 156 & 1.0000 & 1288.8 \\
\hline
\end{tabular}
stable mass number of $Z$

$$
\begin{aligned}
& (B)_{A} \cong\left(\frac{A}{A_{S}}\right)^{p}\left\{k\left(2 Z m_{u} c^{2}+Z m_{d} c^{2}\right)\right\} \\
& \text { where }\left\{\begin{array}{l}
\text { If }\left(\mathrm{A}<\mathrm{A}_{\mathrm{S}}\right), p=4 / 3, \\
\text { If }\left(\mathrm{A}>\mathrm{A}_{\mathrm{S}}\right), p=2 / 3,
\end{array}\right\}
\end{aligned}
$$

Table 1: $T_{0}$ fit the nuclear binding energy near to stable mass number of $Z$ 


\begin{tabular}{|c|c|c|c|}
\hline 66 & 159 & 1.0000 & 1308.7 \\
\hline 67 & 162 & 1.0000 & 1328.5 \\
\hline 68 & 164 & 1.0000 & 1348.3 \\
\hline 69 & 167 & 1.0000 & 1368.1 \\
\hline 70 & 170 & 1.0000 & 1388.0 \\
\hline 71 & 173 & 1.0000 & 1407.8 \\
\hline 72 & 176 & 1.0000 & 1427.6 \\
\hline 73 & 179 & 1.0000 & 1447.4 \\
\hline 74 & 182 & 1.0000 & 1467.3 \\
\hline 75 & 184 & 1.0000 & 1487.1 \\
\hline 76 & 187 & 1.0000 & 1506.9 \\
\hline 77 & 190 & 1.0000 & 1526.8 \\
\hline 78 & 193 & 1.0000 & 1546.6 \\
\hline 79 & 196 & 1.0000 & 1566.4 \\
\hline 80 & 199 & 1.0000 & 1586.2 \\
\hline 81 & 202 & 1.0000 & 1606.1 \\
\hline 82 & 205 & 1.0000 & 1625.9 \\
\hline 83 & 208 & 1.0000 & 1645.7 \\
\hline 84 & 211 & 1.0000 & 1665.6 \\
\hline 85 & 214 & 1.0000 & 1685.4 \\
\hline 86 & 217 & 1.0000 & 1705.2 \\
\hline 87 & 220 & 1.0000 & 1725.0 \\
\hline 88 & 223 & 1.0000 & 1744.9 \\
\hline 89 & 227 & 1.0000 & 1764.7 \\
\hline 90 & 230 & 1.0000 & 1784.5 \\
\hline 91 & 233 & 1.0000 & 1804.4 \\
\hline 92 & 236 & 1.0000 & 1824.2 \\
\hline 93 & 239 & 1.0000 & 1844.0 \\
\hline 94 & 242 & 1.0000 & 1863.8 \\
\hline 95 & 245 & 1.0000 & 1883.7 \\
\hline 96 & 248 & 1.0000 & 1903.5 \\
\hline 97 & 252 & 1.0000 & 1923.3 \\
\hline 98 & 255 & 1.0000 & 1943.1 \\
\hline 99 & 258 & 1.0000 & 1963.0 \\
\hline 100 & 261 & 1.0000 & 1982.8 \\
\hline
\end{tabular}

\section{To understand the discrete behavior and the total energy of electron in hydrogen at- om}

Step-1: To understand the discrete behavior

From Bohr's theory of Hydrogen atom (N.Bohr 1913) maximum number of electrons that can be accommodated in any principal quantum shell are $\left(2 n^{2}\right)$ where $n=1,2,3, .$. this proposal can be reinterpreted as follows: In Hydrogen atom, in $n^{\text {th }}$ principal quantum shell, electron can exist in $\left(n^{2}\right)$ different quantum states. It can be understood as follows. Guess that currently believed s- shell is the basic unit of all quantum shells and it constitutes a maximum of 2 numbers of electrons. With reference to the current concept of $\left(2 n^{2}\right)$ electrons, there can exit $\left(n^{2}\right)$ number of s-shells. If one s-shell represents on quantum state, then with reference to $\left(n^{2}\right)$ number of s-shells, one can expect $\left(n^{2}\right)$ number of different quantum states with different energy levels.

Step-2: To understand the potential energy of $\left(n^{2}\right)$ different states

Let potential energy of electron at any one quantum state be:

$$
\left(E_{\text {pot }}\right)_{n} \cong-\frac{e^{2}}{4 \pi \varepsilon_{0} r_{n}}
$$

Where $r_{n}$ is the distance between electron and proton corresponding to $n^{\text {th }}$ quantum state. Potential energy of possible $\left(n^{2}\right)$ quantum states can be:

$$
\left.\begin{array}{rl}
\in_{\mathrm{pot}} & \cong-n^{2}\left(E_{\mathrm{pot}}\right)_{n} \cong-n^{2}\left(\frac{e^{2}}{4 \pi \varepsilon_{0} r_{n}}\right) \\
& \cong-\left(\frac{\hbar c}{G_{A} m_{e}^{2}}\right)^{2} \frac{\sqrt{m_{p} m_{e}}}{2}
\end{array}\right\}
$$

Based on the Virial theorem (Celso L. Ladera et al 2010) in a central force field, quantitatively kinetic energy is half the potential energy. Following this idea, total kinetic energy of electron for $\left(n^{2}\right)$ quantum state can be:

$\epsilon_{\text {kin }} \cong \frac{1}{2}\left|\epsilon_{\text {tot }}\right| \cong\left(\frac{\hbar c}{G_{A} m_{e}^{2}}\right)^{2} \frac{\sqrt{m_{p} m_{e}} c^{2}}{4}$

Thus, total energy of electron for $\left(n^{2}\right)$ quantum states can be:

$\left.\begin{array}{l}\epsilon_{\mathrm{tot}} \cong \epsilon_{\mathrm{pot}}+\epsilon_{\mathrm{kin}} \\ \cong-\left(\frac{\hbar c}{G_{A} m_{e}^{2}}\right)^{2} \frac{\sqrt{m_{p} m_{e}}{ }^{2}}{4}\end{array}\right\}$

If so, potential energy of electron at any one quantum state can be:

$$
\left.\begin{array}{rl}
\left(E_{\mathrm{pot}}\right)_{n} \cong-\frac{\epsilon_{\mathrm{pot}}}{n^{2}} \cong-\left(\frac{\hbar c}{G_{A} m_{e}^{2}}\right)^{2} \frac{\sqrt{m_{p} m_{e}} c^{2}}{2\left(n^{2}\right)} \\
\cong-\left(\frac{\hbar c}{G_{A} m_{e}^{2}}\right)^{2} \frac{\sqrt{m_{p} m_{e}}{ }^{2}}{2 n^{2}}
\end{array}\right\}
$$

Kinetic energy of electron at any one quantum state can be:

$$
\left.\begin{array}{l}
\left(E_{\mathrm{kin}}\right)_{n} \cong \frac{\in_{\mathrm{kin}}}{n^{2}} \\
\cong\left(\frac{\hbar c}{G_{A} m_{e}^{2}}\right)^{2} \frac{\sqrt{m_{p} m_{e}} c^{2}}{4 n^{2}}
\end{array}\right\}
$$

Total energy of electron at any one quantum state can be:

$$
\left.\left(E_{\text {tot }}\right)_{n} \cong \frac{\epsilon_{\text {tot }}}{n^{2}} \cong-\left(\frac{\hbar c}{G_{A} m_{e}^{2}}\right)^{2} \frac{\sqrt{m_{p} m_{e}} c^{2}}{4 n^{2}}\right\}
$$

Step-3: To understand the emitted photon energy

With reference to the jumping nature of electron from one quantum state to another quantum sate, emitted photon energy can be:

$E_{\text {photon }} \cong\left(\frac{\hbar c}{G_{A} m_{e}^{2}}\right)^{2} \frac{\sqrt{m_{p} m_{e}} c^{2}}{4}\left(\frac{1}{n_{1}^{2}}-\frac{1}{n_{2}^{2}}\right)$

Where $n_{1}=1,2,3, \ldots$ and $n_{2}>n_{1}$.

\section{Conclusion}

So far no model succeeded in coupling and understanding the unified concepts of gravity, electromagnetic and strong interac- 
tions. Based on the proposed concepts and accurate relations and with further research and analysis, different models of final unification can be developed with different proportionality ratios and finally a unified model can be standardized. The absolute magnitude of $G$ can be fixed and uncertainty in its current recommended magnitude can be minimized

\section{Acknowledgements}

The first author is indebted to professor K. V. Krishna Murthy, Chairman, Institute of Scientific Research on Vedas (I-SERVE), Hyderabad, India and Shri K. V. R. S. Murthy, former scientist IICT (CSIR) Govt. of India, Director, Research and Development, I-SERVE, for their valuable guidance and great support in developing this subject.

\section{References}

[1] P. A. M. Dirac, The cosmological constants. Nature, 139, 323, (1937). http://dx.doi.org/10.1038/139323a0.

[2] Witten, Edward. Search for a realistic Kaluza-Klein theory. Nuclear Physics B 186 (3): 412- 428. (1981) http://dx.doi.org/10.1016/05503213(81)90021-3.

[3] David Gross, Einstein and the search for Unification. Current science, Vol. 89, No. p 12. (2005).

[4] Abdus Salam. Einstein's Last Dream: The Space -Time Unification of Fundamental Forces, Physics News, 12(2):36, (1981).

[5] Salam A. and Sivaram C. Strong Gravity Approach to QCD and Confinement. Mod. Phys. Lett., v. A8 (4), 321-326. (1993) http://dx.doi.org/10.1142/S0217732393000325.

[6] Recami E. Elementary Particles as Micro-Universes, and "Strong Black-holes": A Bi-Scale Approach to Gravitational and Strong Interactions. Preprint NSF-ITP-02-94. Posted in the arXives as the e-print physics/0505149, and references therein.

[7] Dine, Michael. Supersymmetry and String Theory: Beyond the Standard Model. Cambridge University Press. (2007) http://dx.doi.org/10.1017/CBO9780511618482.

[8] Roberto Onofrio. On Weak Interactions as Short-Distance Manifestations of Gravity. Modern Physics Letters A, Vol. 28, No. 7 (2013) 1350022 http://dx.doi.org/10.1142/S0217732313500223.

[9] U. V. S. Seshavatharam and S. Lakshminarayana. Two Background Unified Numbers \& Their Possible Role. Prespace time journal, Vol 5, issue 13, pp1338- 1353. (November 2014).

[10]U. V. S. Seshavatharam and S. Lakshminarayana. On the plausibility of final unification with Avogadro number. Prespace time journal, Vol 5, issue 10, pp1028-1041. (2014)

[11]U. V. S. Seshavatharam and S. Lakshminarayana. Nu-cleus in Strong nuclear gravity. Proceedings of the DAE Symp. On Nucl. Phys. 56 $302,2011$.

[12]U. V. S. Seshavatharam and S. Lakshminarayana, Role of Avogadro number in grand unification. Hadronic Journal. Vol-33, No 5, (2010) October. $\mathrm{p} 513$.

[13]U. V. S. Seshavatharam and S. Lakshminarayana, to confirm the existence of atomic gravitational constant. Hadronic journal, Vol-34, No 4, p379 (2011)

[14]U. V. S. Seshavatharam and S. Lakshminarayana. Logic behind the Squared Avogadro number and SUSY. International Journal of Applied and Natural Sciences. Vol. 2, Issue 2, 23-40 (2013).

[15]U. V. S. Seshavatharam and S. Lakshminarayana. Integral charge SUSY in Strong nuclear gravity. Pro-ceedings of the DAE Symp. On Nucl. Phys. 56 (2011) p.842.

[16]U. V. S. Seshavatharam and S. Lakshminarayana. Super Symmetry in Strong and Weak interactions. Int. J. Mod. Phys. E, Vol.19, No.2, (2010), p.263-280. http://dx.doi.org/10.1142/S021830131001473X.

[17]U. V. S. Seshavatharam and S. Lakshminarayana. SUSY and strong nuclear gravity in (120-160) GeV mass range. Hadronic journal, Vol34, No 3, (2011) June, p.277-300.

[18]U. V. S. Seshavatharam and S. Lakshminarayana. New concepts and semi empirical fittings in understanding SUSY and the four cosmological interactions. Prespace time journal, Vol 4, issue 11, (December 2013), pp1027- 1038

[19]Roger Penrose. Chandrasekhar, Black Holes, and Sin-gularities. J. $\begin{array}{lllll}\text { Astrophys. } & \text { Astr. } & \text { (1996) } & \text { 17, }\end{array}$ http://dx.doi.org/10.1007/BF02702305.

[20]Subrahmanyan Chandrasekhar. On Stars, Their Evolu-tion and Thei Stability',Nobel Prize lecture, December 8, 1983.
[21]G.J. Stoney, On the Physical Units of Nature. Phil.Mag. 11 (1881) 381-390. http://dx.doi.org/10.1080/14786448108627031.

[22]P.J. Mohr, B.N. Taylor, and D.B. Newell. CODATA Recommended Values of the Fundamental Physical Con-stants: 2010" by in Rev. Mod. Phys. 84, 1527 (2012) http://pdg.lbl.gov/2014/reviews/rpp2014rev-phys-constants.pdf.

[23]B. Andreas et al. An accurate determination of the Avo-gadro constant by counting the atoms in a $28 \mathrm{Si}$ crystal. Phys. Rev. Let. 106(3):030801 http://dx.doi.org/10.1103/PhysRevLett.106.030801.

[24]B P Leonard. On the role of the Avogadro constant in redefining SI units for mass and amount of substance. Metrologia 44, 82-86 (2007) http://dx.doi.org/10.1088/0026-1394/44/1/012.

[25] K.A. Olive et al. (Particle Data Group), Chin. Phys. C, 38, 090001 (2014) http://dx.doi.org/10.1088/1674-1137/38/9/090001.

[26] Geiger $\mathrm{H}$ and Marsden E. On a diffuse reaction of the particles. Proc. Roy. Soc., Ser. An 82: 495-500, (1909). http://dx.doi.org/10.1098/rspa.1909.0054

[27]Michael O. Distler, Jan C. Bernauer, Thomas Walche. The RMS Charge Radius of the Proton and Zemach Moments. Phys. Lett.B. 696: 343-347, (2011). http://dx.doi.org/10.1016/j.physletb.2010.12.067.

[28] Roberto Onofrio. Proton radius puzzle and quantum gravity at the Fermi scale. EPL, 104 (2013) 20002. http://dx.doi.org/10.1209/02955075/104/20002.

[29] G. Rosi, F. Sorrentino, L. Cacciapuoti, M. Prevedelli and G. M. Tino1 Precision measurement of the Newtonian gravitational constant using cold atoms. Nature 510, 518-521. (2014) http://dx.doi.org/10.1038/nature13433.

[30]L.L. Williams. Analytical Expressions for the Gravita-tional Constant. (August 2009) http://www.konfluence.org/CalculatingG.pdf.

[31]George T Gillies. The Newtonian gravitational constant: recent measurements and related studies. Rep. Prog. Phys. 60 151, (1997) http://dx.doi.org/10.1088/0034-4885/60/2/001.

[32]J Stuhler, M Fattori, T Petelski and G M Tino. MAGIA using atom interferometry to determine the Newtonian gravitational constant. J. Opt. B: Quantum Semiclass. Opt. 5 (2003) S75-S81. http://dx.doi.org/10.1088/1464-4266/5/2/361.

[33] Terry Quinn, Harold Parks, Clive Speake and Richard Davis. An uncertain big G. Phys.Rev. Lett. 112.068103. (2013)

[34]J. B. Fixler; G. T. Foster; J. M. McGuirk; M. A. Kase-vich. Atom Interferometer Measurement of the Newto-nian Constant of Gravity, $\begin{array}{lllll}\text { Science } & 315 & \text { (5808): } & \text { 74-77, } & \text { (2007). }\end{array}$ $\mathrm{http}: / / \mathrm{dx}$.doi.org/10.1126/science.1135459.

[35] Brandenburg, J.E. Unification of Gravity and Electro-magnetism in the Plasma Universe. IEEE Transactions on Plasma Science, v20, n6, p944. (1992)

[36]Jun Luo and Zhong-Kun Hu. Status of measurement of the Newtonian gravitational constant G. Class. Quantum Grav. 17 (2000) 2351-2363 http://dx.doi.org/10.1088/0264-9381/17/12/307.

[37] St. Schlamminger E.Holzschuh, W. Kundig. Deter-mination of the Gravitational Constant Using a Beam Balance. (July 2002) http://www.schlammi.com/pdf/prl02.pdf.

[38]A.V. Manohar and C.T. Sachrajdahttp Quark masses. Updated Jan 2014. Pages 1-20. (pdg.lbl.gov/2014/reviews/rpp2014-rev-quarkmasses.pdf).

[39]Halzen, F.; Martin, A. D. Quarks and Leptons: An In-troductory Course in Modern Particle Physics. John Wiley \& Sons. (1984).

[40]G. Rosi, F. Sorrentino, L. Cacciapuoti, M. Prevedelli and G. M. Tino1 Precision measurement of the Newtonian gravitational constant using cold atoms. Nature 510, 518-521. (2014) http://dx.doi.org/10.1038/nature13433.

[41] Chowdhury, P.R. et al. Modified Bethe-Weizsacker mass formula with isotonic shift and new driplines. Mod. Phys. Lett. A20 p.16051618. (2005) http://dx.doi.org/10.1142/S021773230501666X.

[42]W.D. Myers and W.J. Swiatecki. Table of Nuclear Masses according to the 1994 Thomas-Fermi Model. LBL-36803. (1994).

[43]G. Audi and A.H. Wapstra. The 1993 atomic mass evo-lution. (I) Atomic mass table. Nuclear physics, A 5 65, p1-65 (1993). http://dx.doi.org/10.1016/0375-9474(93)90024-R.

[44]N. Bohr. On the Constitution of Atoms and Molecules. (Part-1) Philos. Mag. 26, 1913, p 1. http://dx.doi.org/10.1080/14786441308634955.

[45]N. Bohr. On the Constitution of Atoms and Molecules. Systems containing only a Single Nucleus. (Part-2) Philos. Mag. 26, 476, (1913) http://dx.doi.org/10.1080/14786441308634993.

[46] Celso L. Ladera, Eduardo Aloma, Pilar Leon. The Virial Theorem and its applications in the teaching of Modern Physics. Lat. Am. J. Phys. Educ. Vol. 4, No. 2, May 2010. 